\title{
Optical and Morphological Characterization of BaSeThinFilms Synthesized via Chemical Bath Deposition
}

\author{
Md. Salahuddin Mina ${ }^{1}$, Humayun Kabir ${ }^{1}$, M. Mahbubur Rahman ${ }^{1 *}$, \\ Md. Alamgir Kabir ${ }^{1}$, Mashudur Rahaman², Muhammad Shahriar Bashar ${ }^{2}$, \\ Md. Saidul Islam ${ }^{2}$, Afrina Sharmin ${ }^{2}$, Farid Ahmed ${ }^{1}$ \\ ${ }^{1}$ Department of Physics, Jahangirnagar University, Savar, Dhaka, Bangladesh. \\ ${ }^{2}$ Institute of Fuel Research and Development, Bangladesh Council of Scientific and Industrial Research, \\ Dhaka, Bangladesh. \\ Corresponding Author's Email:mahbub235@yahoo.com
}

\begin{abstract}
Barium selinide (BaSe) thin films were deposited onto glass substrate via chemical bath deposition (CBD) method. The effect of deposition time on the thin film formation mechanism has been studied to understand the optimum conditions for synthesis process. The phase identification and surface morphology of thin coated films were investigated by X-ray diffraction (XRD) and scanning electron microscopy (SEM), respectively while the optical characterizations were conducted by means of ultraviolet visible (UV-Vis) spectroscopy. XRD study confirms the polycrystalline hexagonal structure of the thin films. The XRD peaks at $2 \theta=23.84^{\circ}$ and $2 \theta=23.86^{\circ}$ showed the preferential orientation along the (021) and (201)plane with deposition time 20 hours and 22 hours respectively, whereas the major peak at (111) was obtained with a deposition time of 24 hours. With the increase in deposition time up to 24 hours, the film gradually grew thicker along with the fine increase in the grain size. The direct optical band gap of the films was measured to be varied from 1.33 to $3.37 \mathrm{eV}$.
\end{abstract}

Keywords:Chemical bath deposition, morphology,X-ray diffraction, optical properties and scanning electron microscopy.

\section{Introduction}

The thin films technology has attracted much attention because of its unique size dependent properties and applications in the optical coating [1], solar cells [2], semiconductor devices [3], optical data storage devices, super capacitor [4], and microwave integrated circuits[5]. In the past few decades, various different techniques such as chemical bath deposition [6], cluster beam deposition[7], electro deposition [8], molecular beam epitaxy [9], thermal evaporation, spray pyrolysis [10], sputter deposition [11], metal organic chemical vapour deposition [12], sol-gel dip-coating technique [13-15], photo-enhanced chemical vapour deposition [16]and plasma polymerization method [17] have been extensively used to synthesize the thin film coatings for commercial and industrial applications. However, in the recent times, chemical bath deposition technique has been widely used for the development of thin film coatings because its cost effectiveness, minimum materials wastage, does not need sophisticated machineries and can be applied in large area deposition at low temperatures. The chemical bath deposition method uses a controlled chemical reaction to deposit a thin film. In the typical experimental approach, the substrates are immersed in solution containing the chalcogenide source, metal ion, and complexing agent.

N.A. Okerekeet al.[18] studied the Tin selenide (SnSe) thin films using CBD technique. Deposition at various deposition conditions was studied in order to investigate the effect of these parameters on the film properties. The structural, morphological and optical properties of films were studied by using $\mathrm{X}$ - ray diffraction (XRD), optical microscopy (OM) and the spectrophotometer. The films prepared were found to be polycrystalline in nature. XRD studied confirms the formation of orthorhombic selenide structure with the preferred orientation along the (210) plane. The material covered the surface of the substrate completely. The indirect optical band gap was found to around1.5 eV. A. Kassimet al. [19] investigated the morphology, composition and optical characterization of $\mathrm{ZnSe}$ thin film. The thin films were deposited on indium tin oxide glass substrate using CBD method. The effect of deposition time (30-120 min) was reported. Thin films prepared at longer deposition time showed better surface coverage compared with small deposition time. The films were confirmed by X-ray diffraction method and energy dispersive analysis of X-ray analysis. The optical band gap from absorption spectra was about 2.55-2.90 eV.N.A. Okerekeet al. [20] investigated the electrical and structural properties of $\mathrm{Ag}_{2} \mathrm{Se}$ thin film developed on glass substrate by $\mathrm{CBD}$ technique and surface of the silver selenide thin films was found to be uniform and exhibits polycrystalline nature. P.U. Asogwaet al. [21] investigated the optical and structural properties of CBD deposited CdSe nanoparticle thin film for photovoltaic application. Analysis of the optical characterization shows that high temperature annealing has significant 
influence on the absorbance, transmittance and band gap energy of the films. From the optical absorption spectra, the band gap energy for CdSe nanoparticle thin films were found to lie within $1.5-1.7 \mathrm{eV}$. XRD studies revealed that the CdSe nanoparticle thin films had a preferred orientation in the $\left(\begin{array}{lll}1 & 0 & 0\end{array}\right)$ plane of a hexagonal structure. The average crystallite size was found to be $23.54 \mathrm{~nm}$. Okerekeet al.[22]investigated the effect deposition time of CBD deposited BaSe thin film. The thin films produced were found to be polycrystalline with cubic structure. The X-ray diffraction peak at $2 \theta=25.8^{\circ}$ shows the preferential orientation along the $\left(\begin{array}{lll}0 & 2 & 1\end{array}\right)$ plane. The band gap value was found to vary from $2.6-2.9 \mathrm{eV}$ with direct transition.From the above review of literatures, it is seen that a lot of interests have been focused on the study of different thin films deposited via chemical bath deposition method for their interesting properties.

In this paper, we focus on the deposition of BaSe thin films using $\mathrm{Na}_{2} \mathrm{~S}_{2} \mathrm{O}_{3}$ as acomplexing agent by chemical bath deposition method. In order to get good quality of thin films, the preparation parameter such as deposition period was optimized and finally to find out the potential applications of the deposited thin films.

\section{A.SAMPLE PREPARATION}

\section{Materials And Methods}

A series of BaSe thin film samples were produced from from $30 \mathrm{ml} 0.5 \mathrm{M}$ barium chloride $\left(\mathrm{BaCl}_{2}\right), 15$ $\mathrm{ml} 0.2 \mathrm{M} \mathrm{Na}_{2} \mathrm{SeO}_{4}$ and $15 \mathrm{ml} 0.2 \mathrm{M}$ sodium thiosulphate $\left(\mathrm{Na}_{2} \mathrm{~S}_{2} \mathrm{O}_{3}\right) . \quad \mathrm{Na}_{2} \mathrm{~S}_{2} \mathrm{O}_{3}$ was used as a complexing agent in order to obtain good quality thin films. $\mathrm{NH}_{3}$ was used to provide an alkaline medium for the growth of the films. All the precursors were mixed together with $60 \mathrm{ml}$ of water to make a solution. In order to form a homogenous solution the mixture was stirred about 2.5 hours. The glass substrates were degreased with $0.1 \mathrm{M}$ ethanol, and etching of the substrates was performed using a $0.1 \mathrm{M} \mathrm{HCl}$ solution. Then the substrates were cleaned ultrasonically with the deionized water for 50 minutes. Then the glass substrates were vertically immersed into the solution and kept the medium undisturbed for various deposition times namely 20, 22, 24 hours etc. The deposited films were tested for adhesion by subjecting it to a steady stream of distilled water.

The phase identification and purity levels of the precursors were confirmed by X-ray diffraction (XRD) experiments. The XRD test of the thin film samples was conducted using a BRUKER D8 XRD system with $\mathrm{Cu}-$ $\mathrm{K}_{\alpha}$ radiation with the wavelength of $1.5406 \AA$, operated at a $40 \mathrm{kV}$ and $40 \mathrm{~mA}$, with high temperature attachment up to $1600^{\circ} \mathrm{C}$. The XRD scan was recorded in the angular range $10^{\circ} \leq 2 \theta \leq 90^{\circ}$ in steps of $0.02^{\circ}$. The surface morphology of the films was investigated using a scanning electron microscope (model: JEOL JSM-6490LA apparatus). The optical properties of the synthesized films were carried out using a UV-Vis spectrophotometer (UV-1601V, Shimadzu Corporation, Japan) while a UV-1200 spectrophotometer (Shimadzu Corporation, Japan) was used to measure the relative reflectance and film thickness using a programmable data pack. Both measurements were done in the wavelength range of $200 \mathrm{~nm}$ to $1100 \mathrm{~nm}$. The absorption data were used to compute the optical band gap of the deposited coatings.

III A. XRD Analysis of the Thin Films

\section{Results And Discussion}

Fig. 1 shows the XRD patterns of BaSe thin films for the deposition periods of 20, 22, and 24 hours. A large number of reflections have been observed from all the samples. The BaSe films deposited with 20 hours shows the (hkl) values 


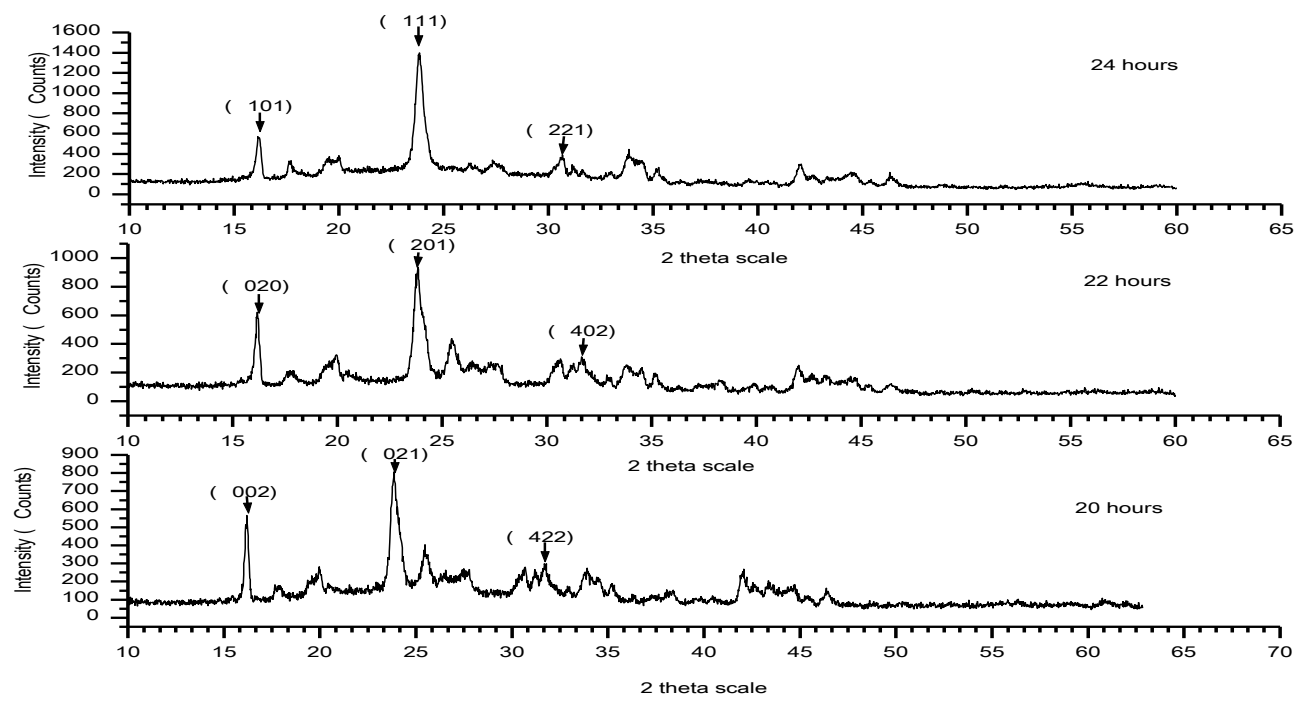

Fig. $1 \mathrm{X}$-ray diffraction patterns of BaSe thin films chemically deposited for a various deposition time.

corresponding to the diffraction peaks of different planes (002), (021) and (422). These planes share the three most prominent reflection having $2 \theta$ values at $\sim 16.2^{\circ}, \sim 23.86^{\circ}$, and $\sim 31.78^{\circ}$ respectively[18]. The samples deposited with 22 hours found to show the diffraction planes of (020), (201) and (402) with $2 \theta$ values of $\sim 16.18^{\circ}, \sim 23.84^{\circ}$, and $\sim 31.66^{\circ}$ respectively while the films with 24 hours of deposition times shows (hkl) planes at (101), (111) and (221) with the three most prominent reflection closes to $2 \theta$ values of $\sim 16.18^{\circ}, \sim 23.87^{\circ}$, and $\sim 30.58^{\circ}$ respectively [22]. According to the diffraction peaks, it has been noticed that all the reflection planes are mixed with odd and even indices. Thus, CBD deposited BaSe thin films are polycrystalline in nature. Further, no diffraction peaks uniquely correspond to metallic barium and selenium were observed. This reveals the fact that the respective highly oriented BaSe crystallites with the (111) planes. It can be seen that the major peaks (111) is strongly dominating the other peaks. XRD studies revealed that the BaSe thin films had a preferred orientation in the (111) plane of a hexagonal structure [21]. The intensity of the peaks of BaSe thin films becomes stronger with increasing the deposition times which confirms the improvement of the crystalline quality of the films due to different deposition times [22].

\section{B. OPTICAL PROPERTIES}

Fig. 2 represents the variation of absorbance, reflectance and transmittance with wavelength of deposited thin films. In the visible region,filmswith 22 hours deposition time show the maximum reflectance of $\sim 16.31 \%$ to $32.08 \%$ and then gradually decreases visible to UV-region. However, the films with 24 hours deposition time shows that the reflectance increases from infrared to visible region. In the visible region, the lowest reflectance was found to be $13.82 \%$ for the samples with 24 hours of deposition times whereas, for the sample with 22 hours deposition times the reflectance was recorded to come down up to $11.15 \%$. Among three films, sample with 20 hours deposition timeexhibits the lowest reflectance of $\sim 9.64 \%$ in the visible range. The overall low reflectance of the sample with 20 hours deposition time makes potential basis for the use as a layer for anti-reflection coating in solar cell production [23]. All deposited filmsshow the maximum transmittance around $364.86 \%$ in the infrared region $(200-300 \mathrm{~nm})$. This high transmittance indicates that the film transmit more energy than it absorbs. The very high transmittance in the visible region for the BaSefilms with 20 hours deposition timemake them useful aesthetic window 

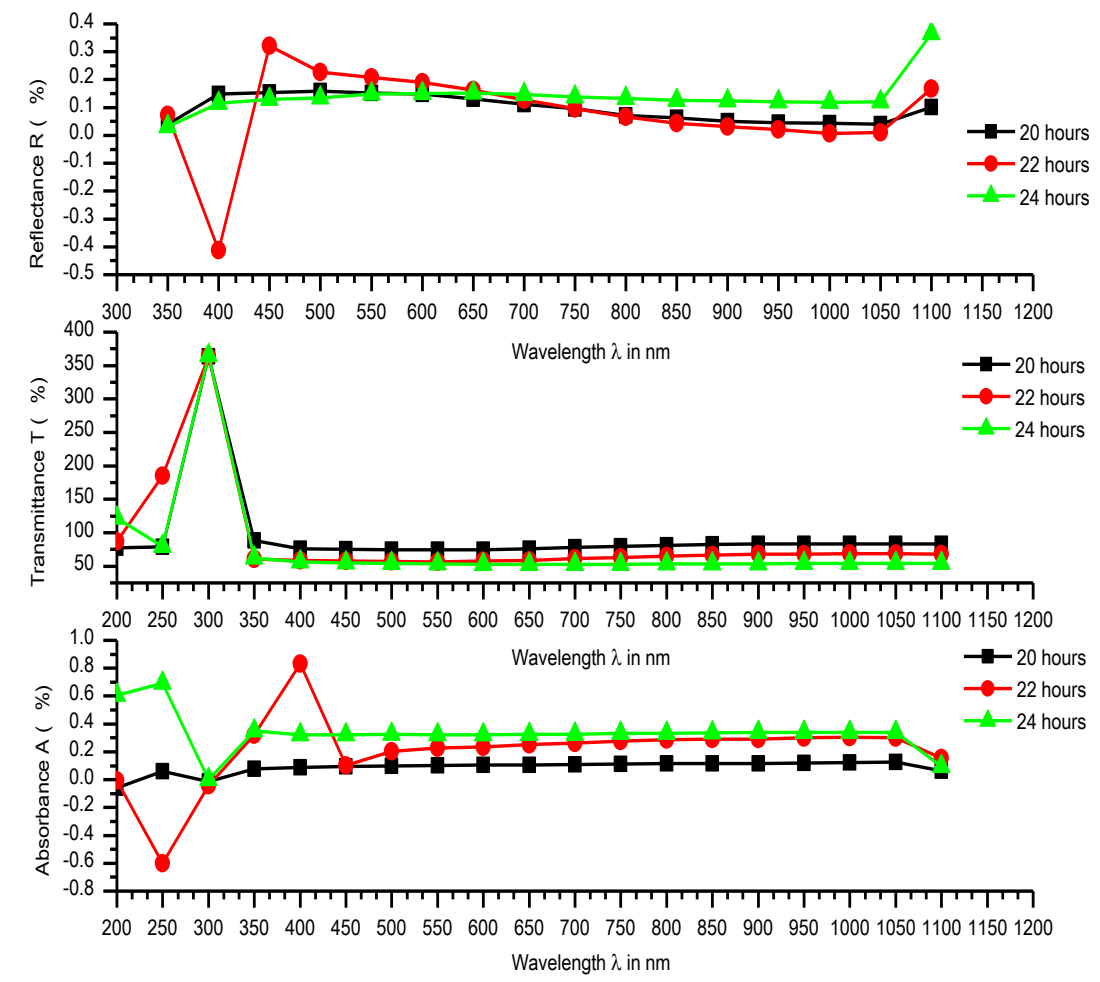

Fig. 2 The optical properties (absorbance, reflectance and transmittance) of deposited films.

glaze material as a p -type layer [24-25]. In the visible region, the highest absorbance of $\sim 32.69 \%$ was recorded for the thin films with 24 hours deposition time. However, in the UV spectrum the absorbance become almost invariant. The films with 22 hours of deposition time show the medium absorbance. In addition, the absorbancewas noticed to be increased in the infrared to visible region. In the UV-region it shows the maximum absorbance $30 \%$. The film with 20 hours of deposition time shows comparatively low absorbance, which wasfound to be increased in the infrared to visible range of solar spectrum. In visible region the maximum absorbance is $10.8 \%$. In UV-region it shows the maximum absorbance $12.16 \%$. It is preferable to use the high absorbance thin film in solar cell. The sample with 24 hours deposition timecan be potentially used as a collector ( $n$ - type layer) in fabrication of solar cell to get maximum output [24].

$\mathrm{The}(\alpha h v)^{2}$ as a function of hv is plotted in Fig. 3 to obtain thedirect energy band gapfor BaSe thin films, where $\alpha$ is the absorption coefficient, $h$ is Planks constant and $v$ is the frequency of light. The direct energy band gap is determined from the intercept of the linear part of the curves extrapolated to zero $\alpha$ in the energy axis. The decrease of band gap with increasing deposition time indicates the improvement of crystalline quality of the deposited film [20]. The decrease of band gap with increasing deposition time is presented in Table1.

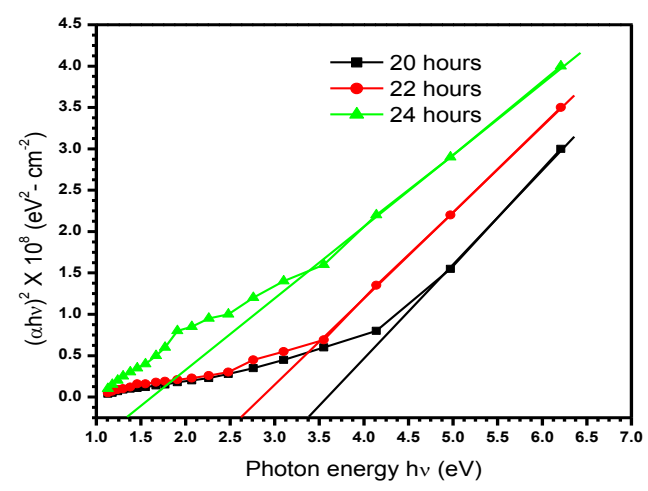

Fig. 3 Determination of direct energy band gap. 


\begin{tabular}{|} 
Table 1: Variation of film thickness and band gap with deposition times. \\
\begin{tabular}{|c|c|c|}
\hline Deposition Time & Film Thickness $(\boldsymbol{\mu m})$ & Band Gap $(\mathbf{e V})$ \\
\hline 20 hours & 1.59 & 3.37 \\
\hline 22 hours & 1.62 & 2.63 \\
\hline 24 hours & 1.64 & 1.33 \\
\hline
\end{tabular}
\end{tabular}

It is found that the band gap of BaSe thin films depend upon the thickness of the films. The increase of film thickness results a decrease in energy band gap of BaSe films. This is true because with increase in films thickness the individual levels of the free atoms will broaden the energy bands and create overlapping levels[22]. This occurs when atoms are brought closer to each other. Hence with high film thickness there are several energy levels resulting in several overlapping energy bands in the band gap of these films. The overlapping energy bands therefore tend to reduce the energy band gap, resulting in lower band gaps for thicker films.Fig. 4 shows the SEM images of the thin films. The grain sizes of the thin films have been investigated at different magnifications. It has been observed that the grain size increases with increasing deposition time [25]. It has been revealed that for the thin films with 20 hours of deposition time, the average grain sizes werefound to be about $12.2 \mu \mathrm{m}$ as shown in Fig. 4 (a). However, when the deposition times were increasedto 22 hours, the average grain sizes wereincreased to $12.7 \mu \mathrm{m}$ as shown in Fig. 4 (b). Further,as soon asthe deposition time wasincreased up to 24 hours, the grain sizes increased to $15.3 \mu$ mas shown in Fig. 4 (c). This indicates that more materials are deposited onto the glass substrate and produce thicker film.

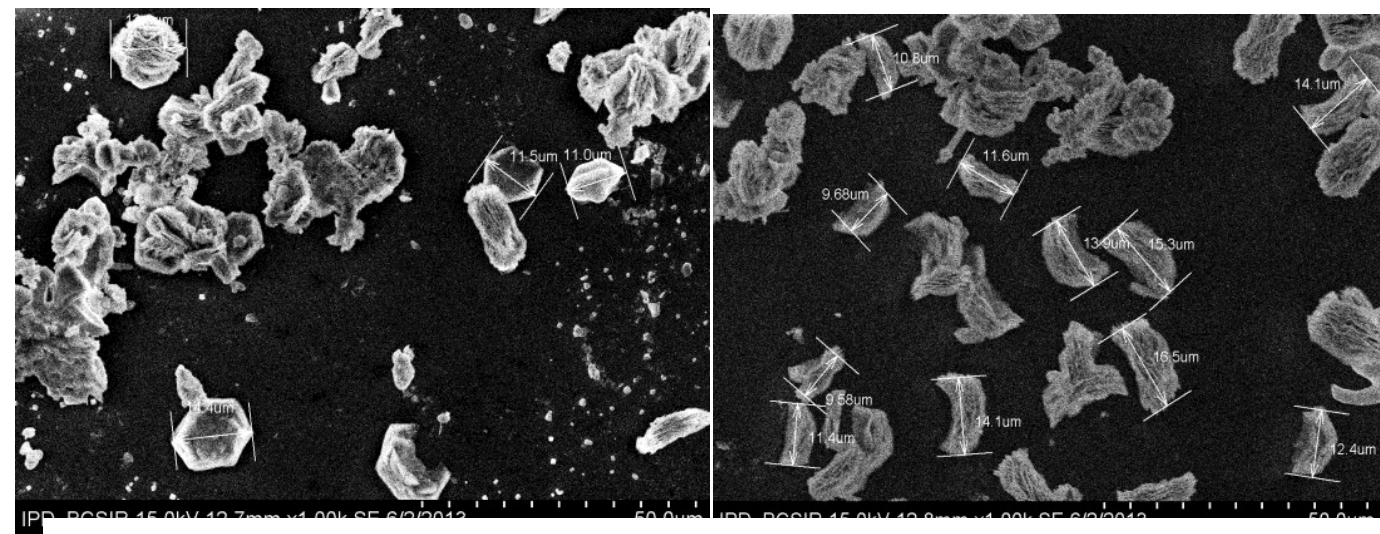

Fig. 4 (a)

Fig. 4 (b)

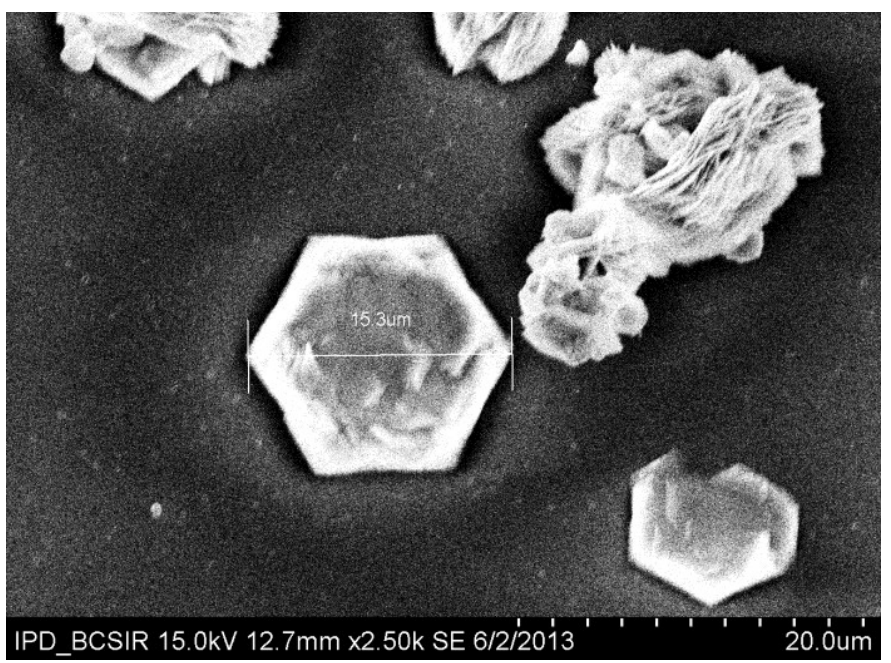

Fig. 4 (c)

Fig. 4 The SEM images of the thin films.

\section{Conclusions}

BaSethin films have been chemically deposited on ultrasonically cleaned glass substrates from aqueous solutions containing $\mathrm{BaCl}_{2}, \mathrm{Na}_{2} \mathrm{SeO}_{4}, \mathrm{Na}_{2} \mathrm{~S}_{2} \mathrm{O}_{3}$ and $\mathrm{NH}_{3}$. XRD study confirms polycrystalline hexagonalstructure of BaSe thin film coatings. The most intense peak of the films was obtained at $2 \theta=23.87^{\circ}$ belongingto the (111) plane. As the deposition period was increased up to 24 hours, the film starts to grow thicker whichcould be seen 
in the SEM images. The SEM image showed that this film has uniform, smaller crystal size and covered the entire substrate surface completely. Absorptance is found to be increased slightly with increase in deposition time. The band gaps are decreased with increase in thickness of the deposited films. The values of direct band gapare found to be in the range 1.33-3.37 eV.Due to high absorptance, the 20 hours deposited thin film is preferable to fabricate the high absorptance thin film for solar cell applications. The films synthesized with 24 hours deposition time can be used as a collector (n- type layer) in fabrication of solar cell to get maximum output.

\section{Acknowledgements}

Authors would like to thank the Department of Physics, Jahangirnagar University, Dhaka, Bangladesh and Institute of Fuel Research and Development (IFRD),Bangladesh Council of Scientific and Industrial Research (BCSIR), Dhaka for providing the laboratory facilities and technical support to carry out this work comfortably.

\section{References}

[1]. K. L. Chopra, Thin Film Phenomena, McGraw Hill, New York, USA (1969).

[2]. J. Pearce, Photovoltaics: A Path to Sustainable Futures, 34 (7), 663-674 (2002).

[3]. R.M. Swanson, Photovoltaics Power Up,324, 891-892 (2009).

[4]. K. Seshan, Hand book of thin film deposition processes and techniques, Intel Corporation Santa Clara, California, USA (2001).

[5]. T.P. Gujar, V.R. Shinde, C.D. Lokhande, R.S. Mane, Sung-Hwan Han, Applied Surface Science,250, 161-167 (2005).

[6]. C.D. Lokhande, Mater. Chem. Phys., 28, 1-10 (1991).

[7]. B.R. Appleton, Ion Beam Processes in Advanced Electronic Materials and Device Technology, Materials Research Society, Pittsburgh,PA, USA (1985).

[8]. Y. Gao, H. Niu, C.Q. Chen, Chem. Phys. Lett., 367, 141-145 (2003).

[9]. A.C. Gossard, Treat. Mater. Sci. Tech.,24, 13-66 (1982).

[10]. V. Popescu, et al., J. Opto. Adv. Mater.,8, 1187-1193 (2006).

[11]. A. Roth, Vacuum Technology, 2nd Ed., North-Holland Pub. Co., Holland (1982).

[12]. S.M. Ojha, Phys. Thin Films,12, 237-296 (1982).

[13]. A. Amri, X.Duan, C.Y. Yin, Z.-T. Jiang, M.M. Rahman, T. Pryor, Appl. Surf. Sci., 275, $127-135$ (2013).

[14]. A. Amri, Z.-T. Jiag, T. Pryor, C.Y. Yin, Z. Xie, N. Mondinos, Surf. Coat. Tech., 207, 367-374 (2012).

[15]. A. Amri, Z.-T. Jiang, P.A. Bahri, C.Y. Yin, X. Zhao, Z. Xie,X. Duan, H. Widjaja, M.M. Rahman, T. Pryor, J. Phys. Chem.C,117, 16457-16467 (2013).

[16]. J.W. Peters, F.L. Gebhart, and T.C. Hall, Solid State Tech., 23(9), 121-126 (1980).

[17]. H. Kabir, M. M. Rahman, T. S. Roy, A.H. Bhuiyan, International Journal of Mechanical \& Mechatronics Engineering, 12 (5), $30-34$ (2012).

[18]. N.A. Okereke, A.J. Ekpunobi, Chalc. Lett.,7 (9), 531-538 (2010).

[19]. A. Kassim, Ho Soon Min,Euro. J. Appl. Sci.,3, 75-80 (2011).

[20]. N.A. Okereke, A.J. Ekpunobi, J. Opto. Bio. Mat.,3(2), 51 - 55 (2011).

[21]. P.U. Asogwa,J. Non-Oxide Glasses,2 (4),183 - 189(2010).

[22]. N.A Okereke, A.J. Ekpunobi,Chalc.Lett.,8 (1)1, 9-14 (2011).

[23]. I.A. Ezenwa, Adv. Appl. Sci. Res., 3 (2), 980-985 (2012).

[24]. F.I. Ezema, A.B.C. Ekwealor\& R.U. Osuj,Turk J. Phys., 30, 157-163(2006).

[25]. A.B. David, Electronic devices and circuits, Fourth edition, Prentice Hall of India (1999). 